\title{
ГРАФОАНАЛИТИЧЕСКИЙ МЕТОД ОПРЕДЕЛЕНИЯ ЗАВИСИМОСТИ ПЕРЕДАТОЧНОГО ОТНОШЕНИЯ МНОГОЗВЕННЫХ АВТОПОЕЗДОВ ПРИ ПОВОРОТЕ НА 90
}

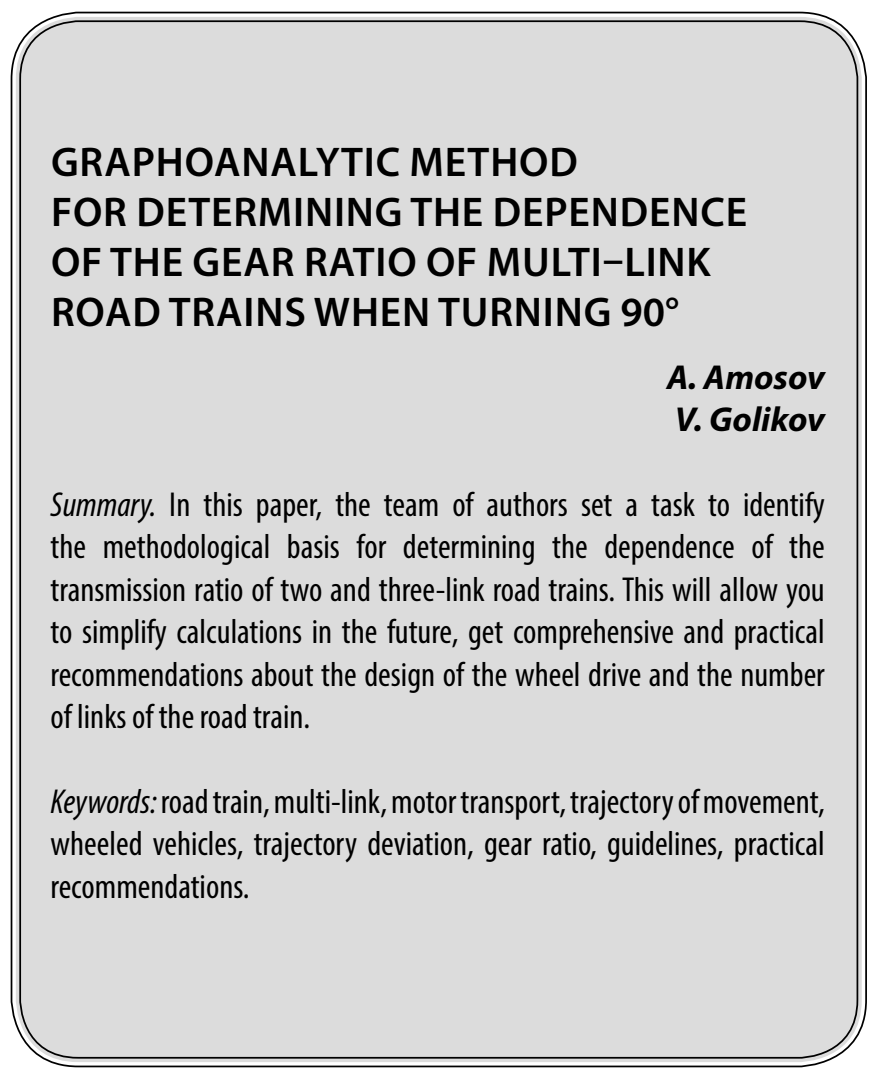

\section{Ввехение}

реди методик, по которым определяют угол поворота колес прицепного звена автопоезда, на сегодняшний день наиболее распространенными являются системы, осуществляющие практическое управление, зависящее от величины угла складывания [1]. При таком управлении, основная характеристика будет выражаться в придаточном отношении $i$, что значит зависимость угла поворота данного колеса полуприцепа относительно угла складывания. Чтобы выбрать величину $i$, относящуюся к колесам прицепного звена, следует изучить условия установившегося движения центров данных колес и его звеньев относительно круговой траектории неизменного радиуса. Для большинства случаев радиус поворота R следует обозначать как 35 м [2-4].

Чтобы найти влияние типа поворота относительно характера изменения траектории полуприцепа был осуществлен расчет траектории звеньев автопоездов из двух и трех звеньев [5] (рисунок 1).
Амосов Алексей Германович

Ассистент, Московский авиационный институт (национальный исследовательский университет) lamosov@yandex.ru

Голиков Владислав Андреевич Ассистент, Московский авиационный институт (национальный исследовательский университет) fordik08@mail.ru

Аннотация: В данной работе коллектив авторов поставил перед собой задачу выявления методической базы для определения зависимости передаточного отношения двух и трехзвенных автопоездов. Это позволит в будущем упростить расчеты, получить комплексные и практические рекомендации по поводу конструкции колесного привода и количества звеньев автопоезда.

Ключевые слова: автопоезд, многозвенность, автотранспортировка, траектория движения, колесные машины, отклонение траектории, передаточное отношение, методические указания, практические рекомендации.

\section{Образец Авижения в повороте на $90^{\circ}$}

За основу тягача автопоезда из двух и трех звеньев определена м. Основой промежуточной тележки автопоезда из трех звеньев считается м. Базовое значение полуприцепов автопоезда из двух и трех звеньев изменчиво. Для всех схем расчета места сцепки звеньев являются тождественными относительно опорных точек соответствующего звена.

Изучим поворот, равный 90. Для наглядности можно увидеть траектории точки опоры тягача и полуприцепов с базами на 2 и 3 рисунках. Опоры соответствуют 20 и 25 м автопоезда из трех звеньев, при разных значениях передаточного отношения.

Основываясь на расчетах, проведенных на рисунках 4 (автопоезд из двух звеньев) и 5 (три звена) были сконструированы зависимости изменения траектории полуприцепов относительно модуля передаточного от- 


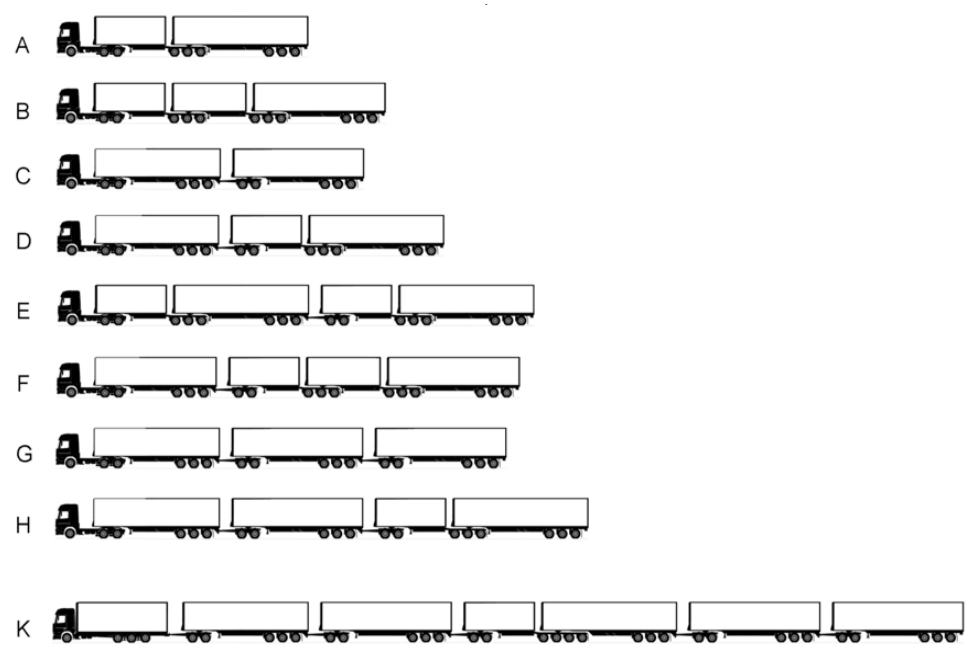

Рис. 1. Многозвенные автопоезда

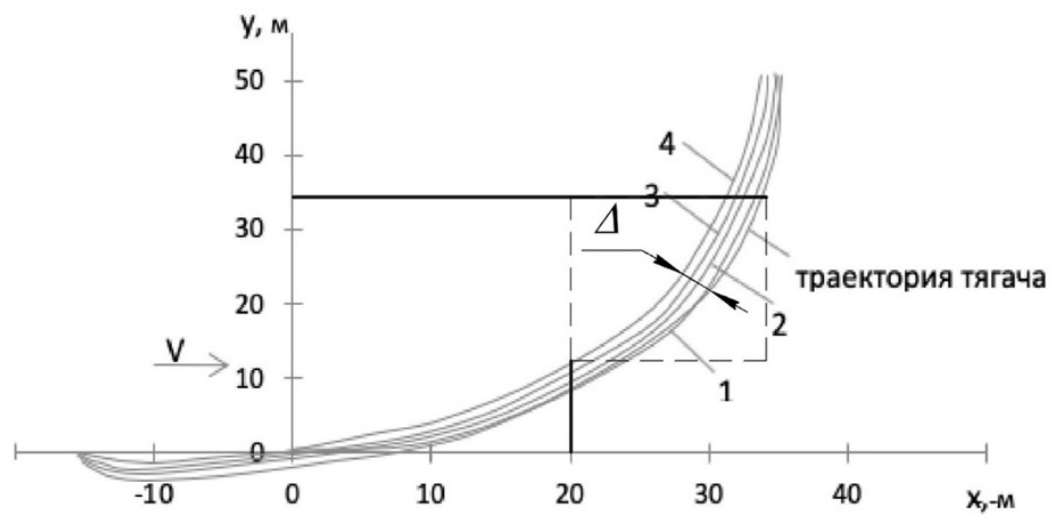

Рис. 2. Траектории тягача и полуприцепа с базой $L_{n}=20$ м и трехзвенног (с базой промежуточной тележки $L_{m}$ ) при различных значениях передаточного отношения і при повороте на $90^{\circ}$ с радиусом $\mathrm{R}=35 \mathrm{M}$

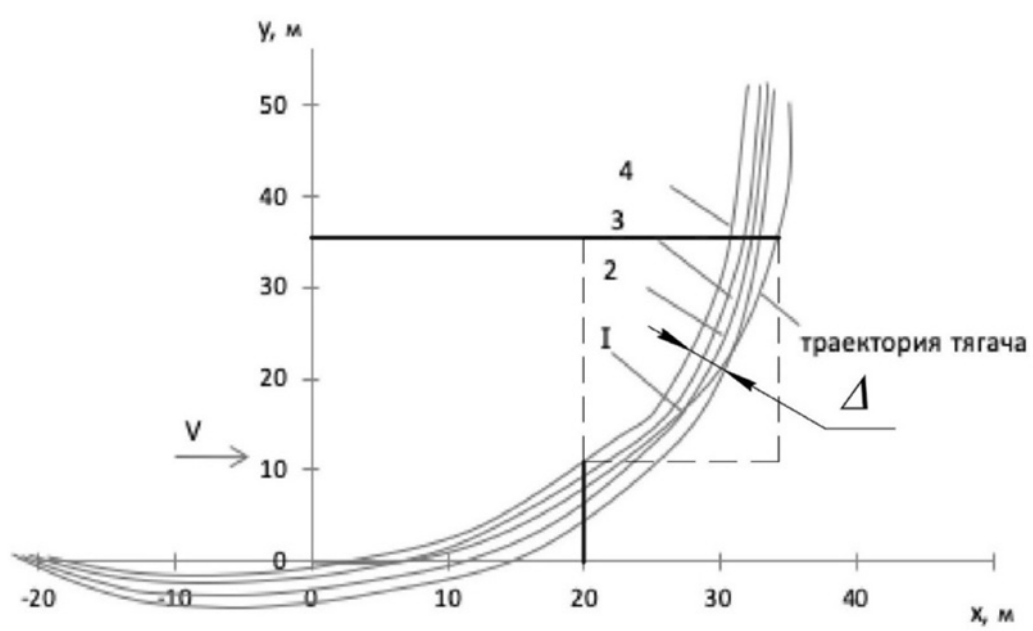

Рис. 3. Траектории тягача и полуприцепа с базой $L_{n}=25$ м трехзвенного (с базой промежуточной тележки $\left.L_{m}\right)$ ) автопоезда при различных значениях передаточного отношения і при повороте на $90^{\circ}$ с радиусом $\mathrm{R}=35 \mathrm{~m}$ 


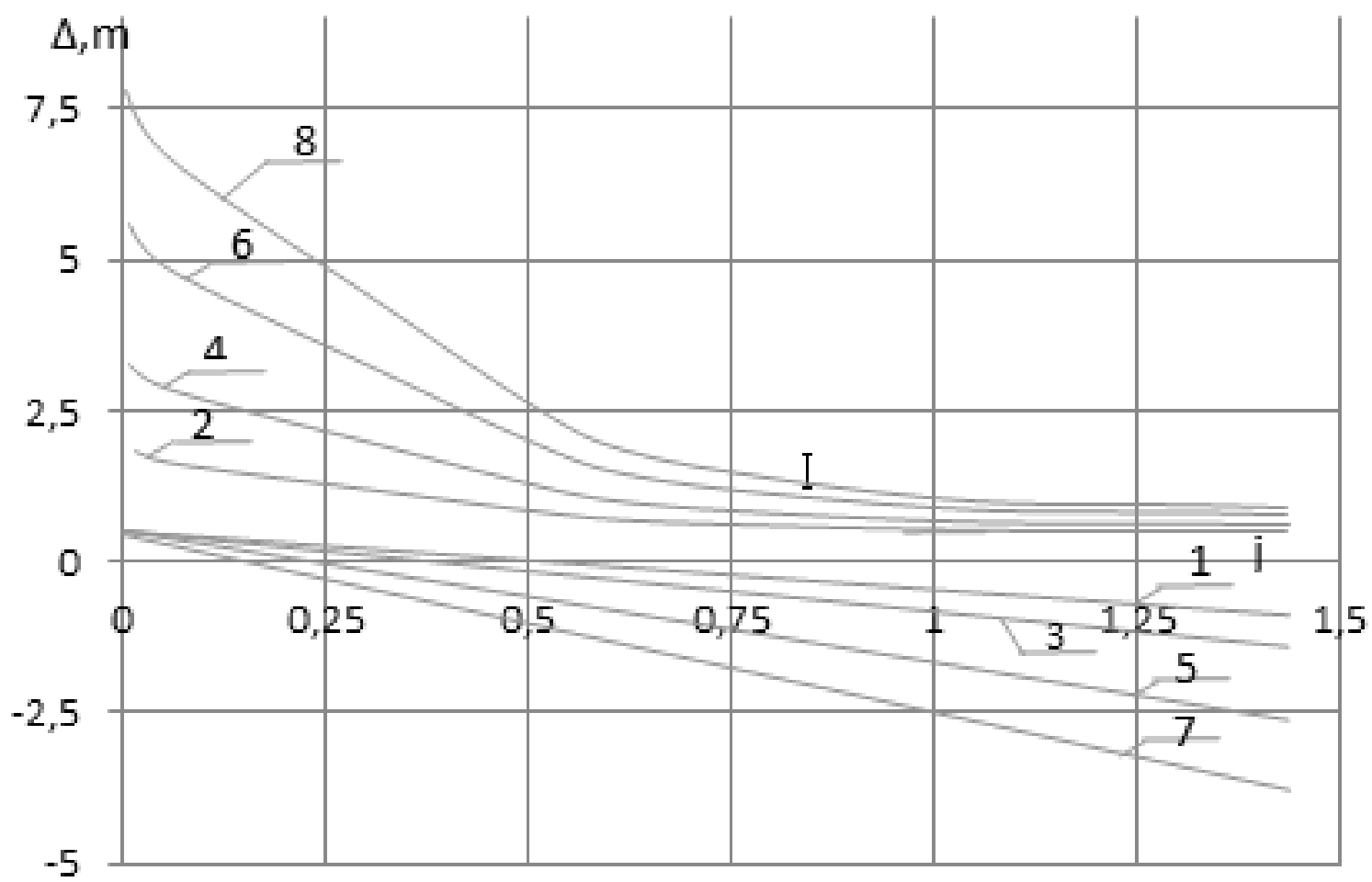

Рис. 4. Смещения траекторий полуприцепа двухзвенного автопоезда от траектории опорной точки при повороте на $90^{\circ}$

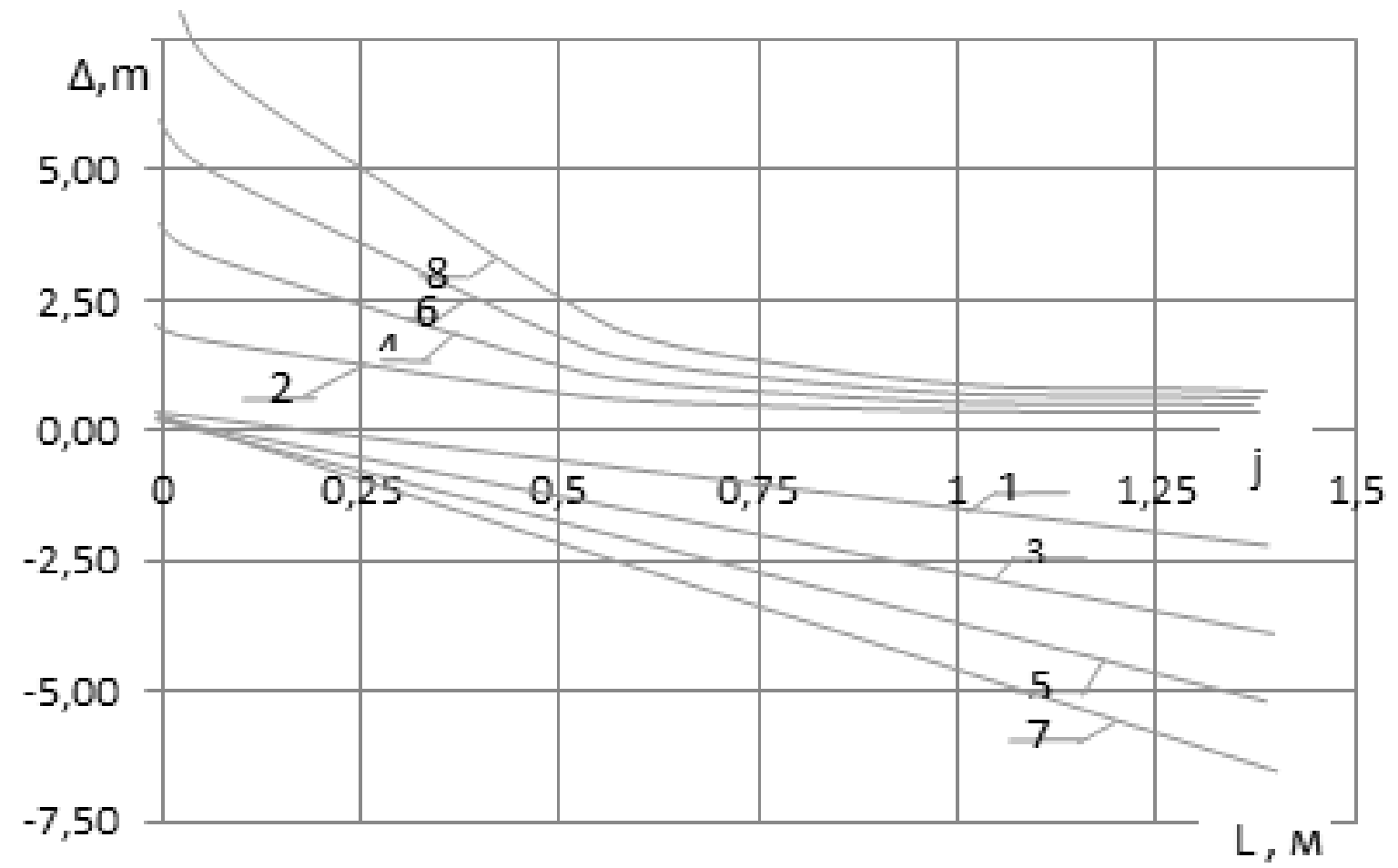

Рис. 5. Смещения траекторий полуприцепа трехзвенного автопоезда от траектории опорной точки в зависимости от передаточного отношения и базы полуприцепа при повороте на $90^{\circ}$ 


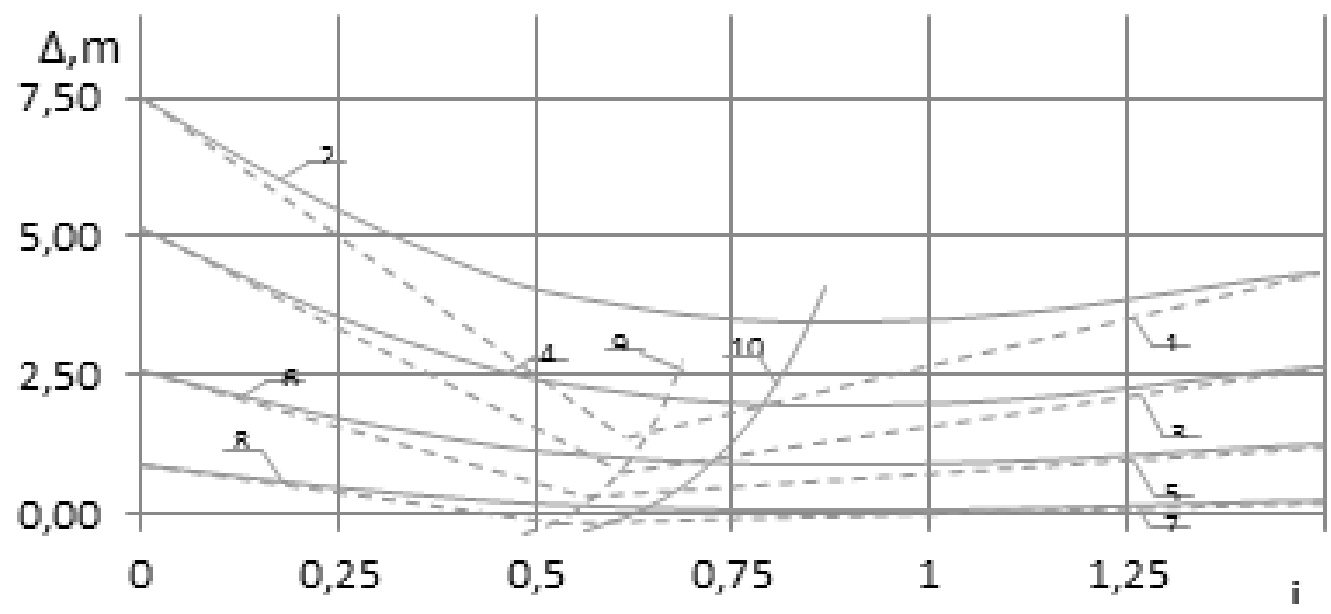

Рис. 6. Максимальные смещения траекторий полуприцепа двухзвенного автопоезда при поворотах на $90^{\circ}$ (с радиусом м)

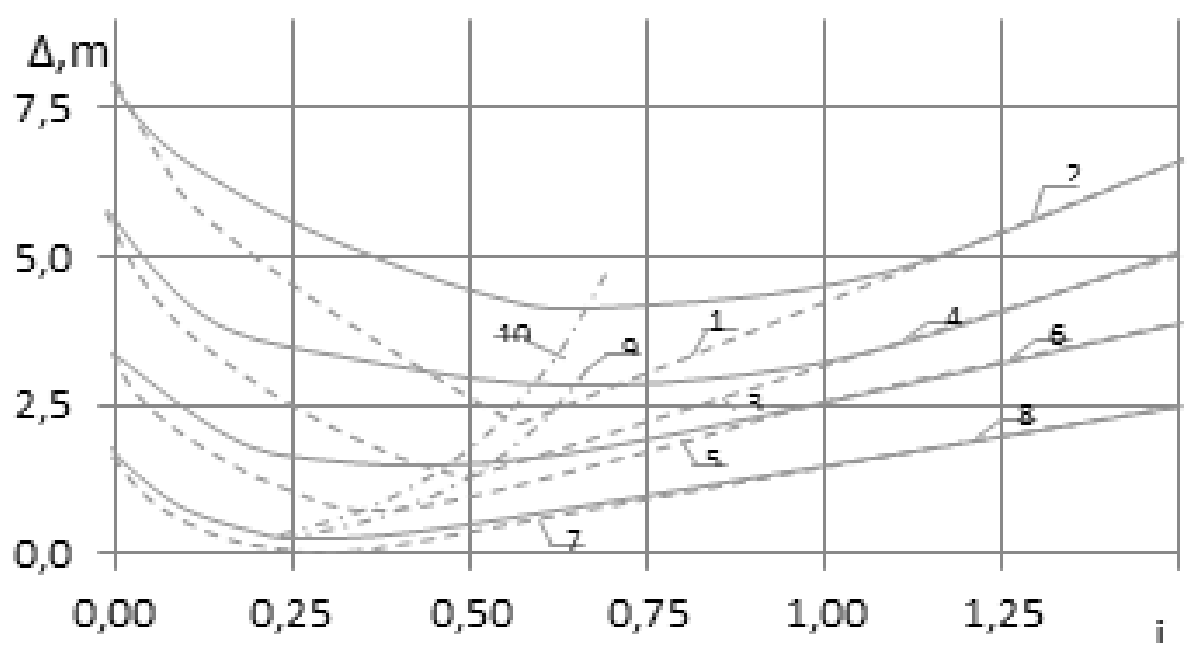

Рис. 7. Максимальные смещения траекторий полуприцепа трехзвенного (с промежуточной тележкой) автопоезда при поворотах на (с радиусом $\mathrm{R}=35 \mathrm{~m}$ )

ношения $|i|$ системы, управляющей поворотом колес для разного значения баз полуприцепов $L_{n}$.

Можно заметить факт, зависимости смещения на входе в поворот от размера модуля придаточного отношения, причем эта зависимость линейного характера. Также важно отметить, что чем меньше значение, тем меньшая величина смещения ему соответствует. Это походит как для автопоезда из двух звеньев, так и для трехзвенного.

Как пример можно рассмотреть рост модуля придаточного отношения системы поворота всех колес полуприцепа, который включен в состав автопоезда из трех звеньев. От 0,5 до 1 смещения, как следствие увеличивается примерно в 2 раза.

Рассматривая полуприцеп, двух и трехзвенный автопоезд, можно заметить, что различие смещений на входе в поворот, равный $90^{\circ}$ лишь количественная. У полуприцепа трехзвенного смещение больше, чем тождественного двухзвенного, имея равное значение базы $L_{n}$ и модуля передаточного отношения.

Как показано на рисунках 4 и 5, ситуация при выходе из поворота, равного $90^{\circ}$ обратна тому, что мы видим при входе. При этом, чем больше становится значение, тем меньше становятся смещения, в соответствии с за- 


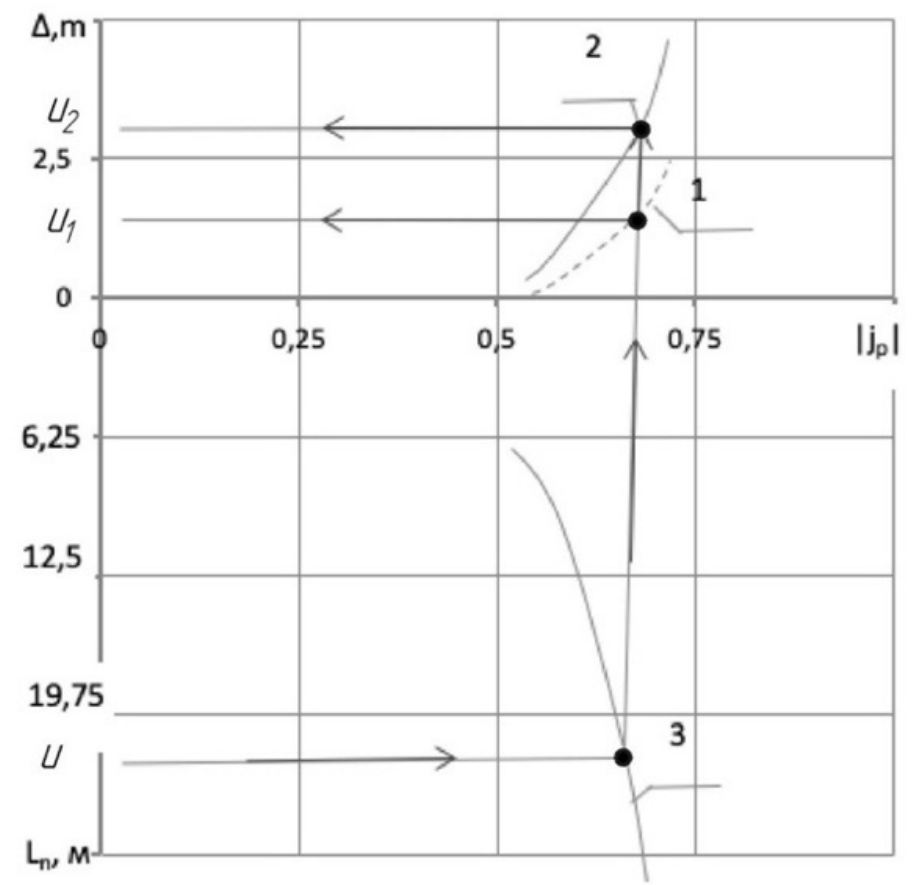

Рис. 8. Номограмма для определения величины передаточного отношения і двухзвенного автопоезда и смещения траектории при заданном значении базы

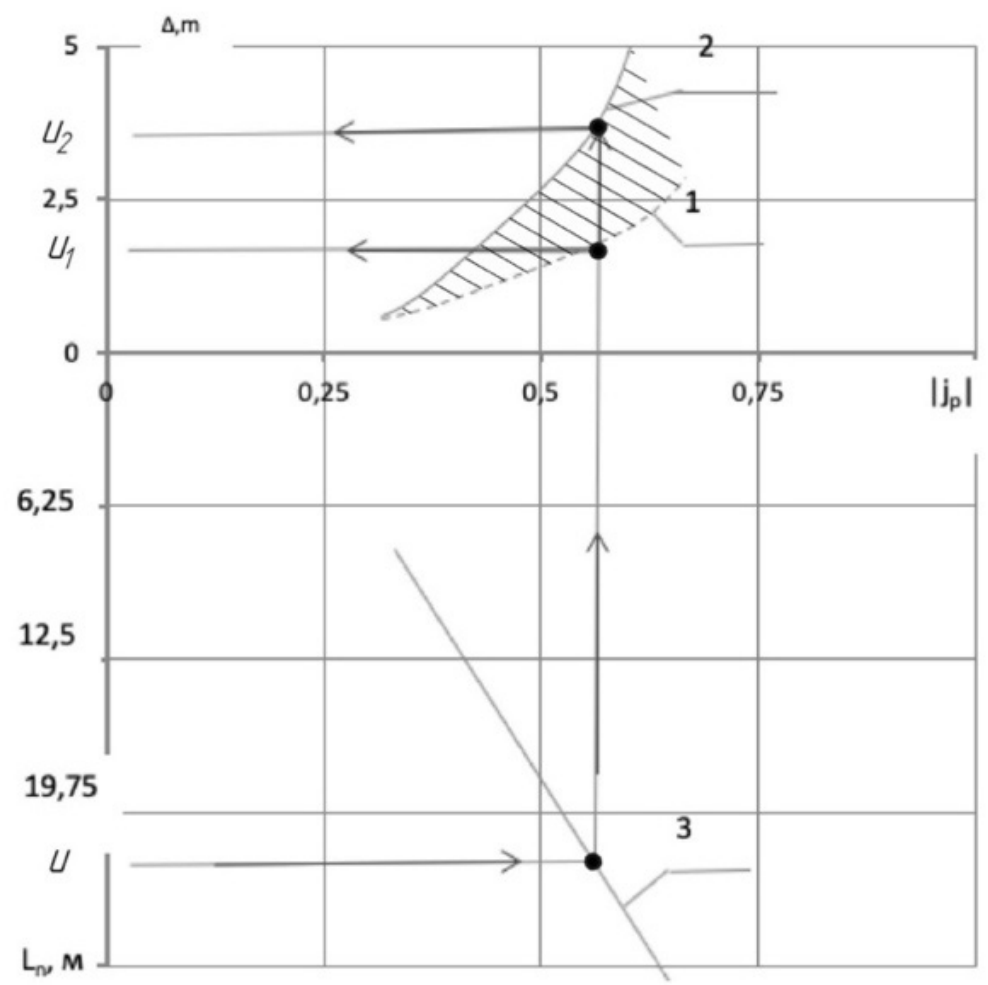

Рис. 9. Номограмма для определения величины передаточного отношения і полуприцепа трехзвенного (база промежуточной тележки) автопоезда и смещения траектории при заданном значении базы 


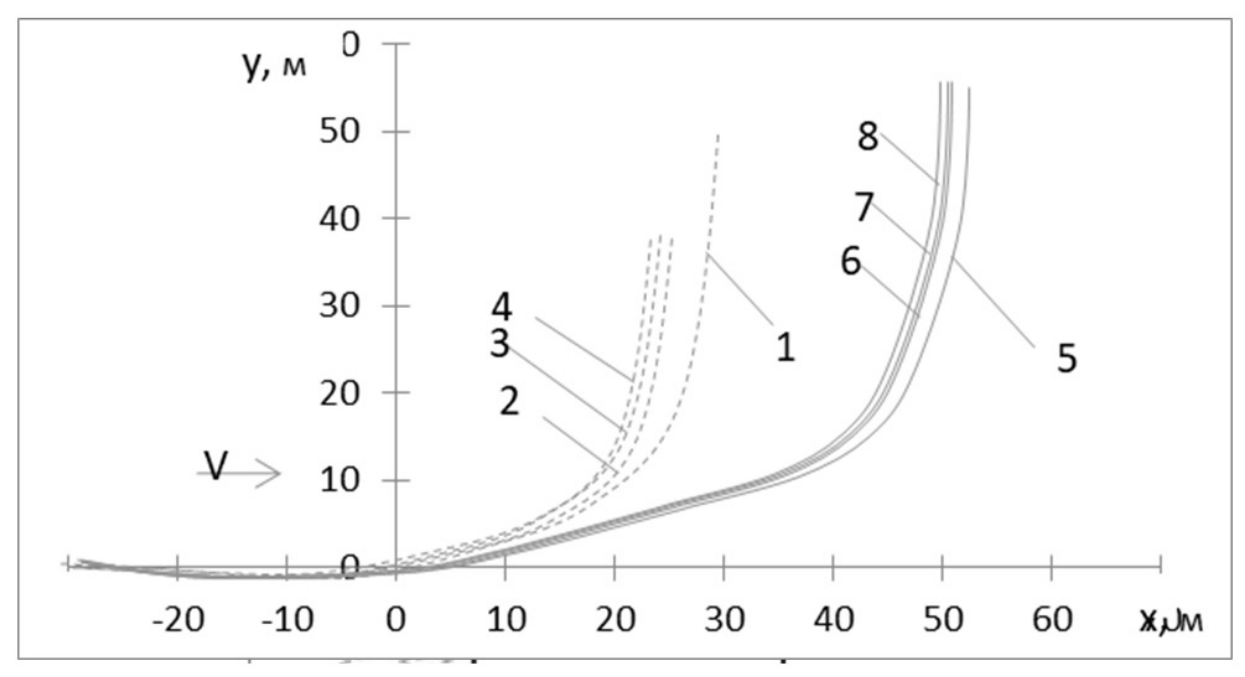

Рис. 10. Траектории тягача и полуприцепа с базой $L_{n}=25$ м трехзвенного автопоезда (с базой промежуточной тележки $\left.L_{m}\right)$ с различными значениями передаточных отношений і при повороте на $90^{\circ}$

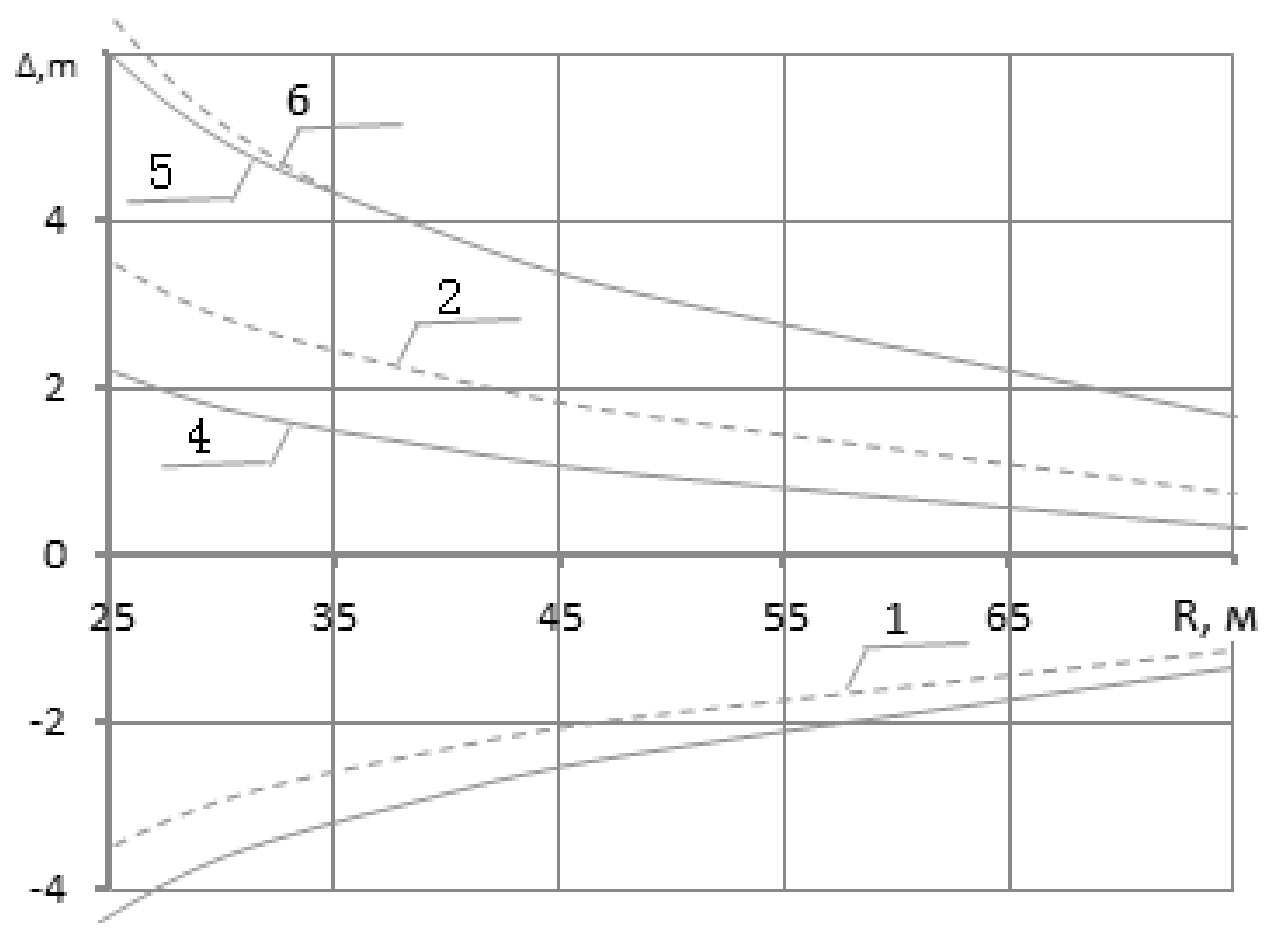

Рис. 11. Смещения траектории полуприцепа (с базой м) трехзвенного (с базой промежуточной тележки) автопоезда в зависимости от радиуса поворота и передаточного отношения на различных участках поворотов на $90^{\circ}$

коном, близком по иду к гиперболическому. При этом, отлично от вхождения в поворот, когда полуприцеп осуществляет «забегание» на внешнюю сторону от траектории тягача, выход из поворота обуславливает смещение внутрь (стремясь к центру), относительно траектории тягача, соответственно.
Максимальная величина смещения на выходе из поворота может наблюдаться, если (колеса, не осуществляющие поворот) полуприцепа. Тождественно со случаем вхождения в поворот, смещение полуприцепа автопоезда из трех звеньев по количеству превышает случаи такого же двухзвенного [6]. 
Следует отдельно обратить внимание на факт того, что от 0 и до конкретного значения передаточного отношения смещения при выходе из поворота имеют большее значение, чем при входе в поворот.

Имея смещения, можно утверждать, что величина при входе и выходе будет одинакова. Однако, если смещения при входе в поворот превышает величину при выходе.

Рисунки 6 и 7 представляют кривые максимальных значений смещений траекторий полуприцепов и автопоездов из двух и трех звеньев. Данные кривые выстроены по следующей схеме. От 0 до за образец максимальных принимаются смещения при выходе из поворота, а при, используются абсолютные величины при выходе.

\section{Обсужление}

Основываясь на максимальных значениях кривых при смещении траектории полуприцепов автопоезда из двух звеньев, при повороте равном 90 была построена номограмма на рисунке 8. Она позволяет определить величину модуля передаточного отношения системы поворота колес полуприцепа и соразмерных смещений траекторий, имея заданное значение при.

Можно заметить, что каждой величине базы полуприцепа соответствуют точки с координатами где придаточные отношения системы поворота колес полуприцепа позволяют минимизировать величину смещений при повороте, равном $90^{\circ}$.

Тем не менее, необходимо определить определенное передаточное отношение, позволяющее агрегату иметь смещения, даже если большего значения, чем минимальное, но не очень далекое от него. В данном случае оптимальным значением в интервале, обеспечивающее рост величины смещения при осуществлении поворотов на $90^{\circ}$.

Значение, которое было найдено, позволяет обеспечить минимальную ширину полотна дороги, которая требуется автопоезду во время движения по криволинейным отрезкам дороги [8].

Схожая номограмма для трехзвенного изображена на рис. 9.

Аналогичную схему можно наблюдать в отношении трехзвенного автопоезда, разница будет заключаться лишь в значении смещения трехзвенного при повороте на $90^{\circ}$, оно уменьшается на 30-35\% (при значении в 60$65 \%$ у двухзвенного) [9].
Для всех изучаемых случаев передаточное отношение полуприцепа колебалось в пределах от $-0,15$ до $-0,8$.

На рисунке 11 изображены смещения траектории полуприцепа, соответствующие двум значениям передаточного отношения: - расчетное значение, которое определяется исходя из условия движения по кругу всех звеньев автопоезда, относительно окружности $\mathrm{R}=35 \mathrm{M}$ и - это значение определяется, основываясь на номограмме, приведенной на рисунке 13.

На рисунке 10 также изображены смещения траектории тягача, зависящие от величины модуля передаточного отношения $|i|$ и радиуса на разных отрезках.

Изучая рисунок 11, можно заметить, при вхождении в поворот, равный 90, а также S-образный поворот, смещения полуприцепа с будут иметь меньшее значение, чем при ориентировочно на $30 \%$. При выходе из поворота, равного $90^{\circ}$ можно наблюдать иную картину. Смещения полуприцепа с превышает такие же, но с, примерно на 50-60\% Однако, это не влияет на требуемое расширение полотна дороги, так как уширения обусловлены максимальными величинами смещений. Смещение же полуприцепа с, при входе в поворот на всем отрезке изучаемых величин радиусов превышают по абсолютной величине соответственные при выходе из поворота [10].

\section{Результат}

Полученный результат базируется на изучении финальных данных и представлен в формате блок-схемы. (рис. 12)

Изучение начинается с организации начальных данных. Этот этап характеризуется сбором базовой информации об объекте использования, сюда входит: функции объекта, сфере его использования, топологические характеристики обстановки и так далее.

После этого начинается процесс предварительной оценки возможности использования метода. Во время этого этапа следует обработать найденную информацию и изучить возможность применения выбранного метода. Положительный результат анализа считается, если есть комплекс результатов, который удовлетворяет требования технического задания. В том случае, если использовать метод невозможно, требуется найти другой способ получить результат.

При получении положительного результата, следует перейти к порядку процессов. Рассмотрим более конкретно: 


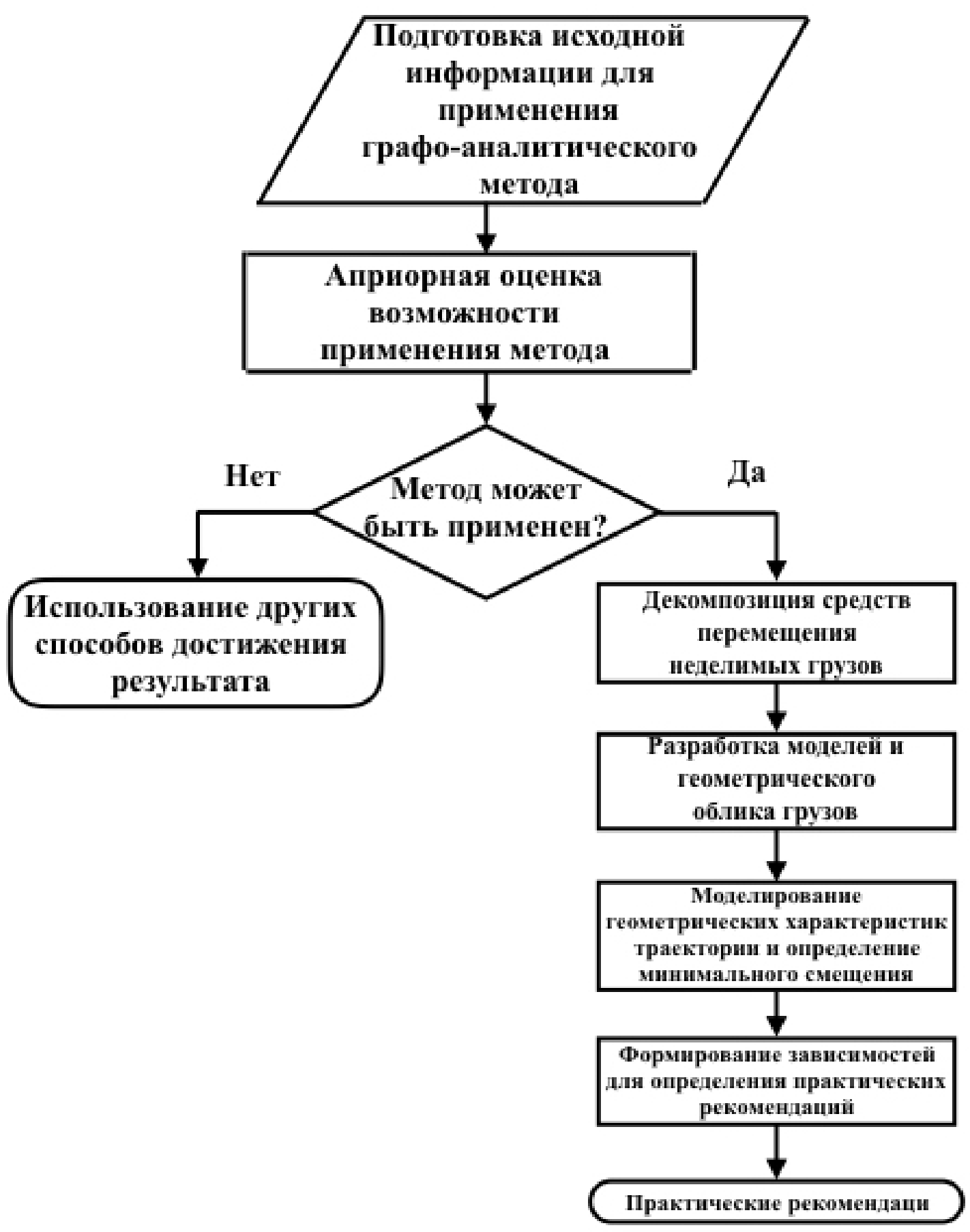

Рис. 12. Блок-схема аналитического метода определения зависимости

Декомпозиция методик перевозки грузов - процесс, который является обязательным для поиска иерархического уровня, к которому применяется метод.

Процесс создания моделей и геометрического вида грузов, позволяющий найти общие обязательные геометрические параметры грузов, не подлежащих разделению.
Создание моделей геометрических параметров траектории и поиск минимальной величины смещения это позволяет найти свойства инфраструктурных границ района транспортировки и выявить критические отрезки следования.

Процесс создания зависимостей для поиск практических рекомендаций является необходимым, чтобы уста- 
новить математические и геометрические связи результатов предшествующих процессов [10].

При выполнении всех действий возможно получение практических рекомендаций по использованию результатов работы.

\section{ВывО $\triangle \mathrm{b}$}

Рассматривая графическую и аналитическую части метода, можно увидеть, что каждый автопоезд, имеющий определенную базу полуприцепа, имеет такую величину передаточного отношения, при котором обеспечивается оптимальные смещения траекторий полуприцепа, с учетом поворотов на $90^{\circ}$.

Смещение траектории полуприцепов, имеющих передаточное отношение, выбранные на основе номограмм, при совершении поворота, равном 90 меньше на 6065\% и 30-35\% для двухзвенного и трехзвенного, имеющего базу промежуточной тележки, соответственно.
Максимальная величина смещения траектории звеньев с передаточными отношениями системы поворота колес полуприцепа, которая была выбрана, основываясь на номограмме, меньше их, при передаточных отношениях, выбранных из кругового движения, если радиусы.

Рассматривая автопоезд из двух звеньев, выбор размера придаточного отношения $i$ из условия движения по кругу всех звеньев автопоезда относительного одного центра поворота, эти смещения были бы равны 2,8 м, 3,6 м, соответственно. Исходя из этого, оптимальный выбор передаточного отношения дает возможность снизить уровень смещения при повороте на $90^{\circ}$ почти на $60-65 \%$.

Кроме этого, знание того, что имеет меньшее значение, чем, выбираемого из условия движения по кругу, позволяет снизить величину угла поворота управляемых колес. Все это приводит к облегчению конструкции колесного хода и делает производство дешевле.

\section{ЛИТЕРАТУРА}

1. Гинцбург, Л.Л. Устойчивость управляемого движения автомобиля относительно траектории / Л.Л. Гинцбург // Автомобильная промышленность.1977.—№ 9.-C. 27-31.

2. ГОСТ 33382-2015 Автомобильные дороги общего пользования. Техническая классификация — М.: Стандартинформ, 2016. — Б/С

3. Маланин, В. В. Определение критических скоростей и радиусов поворота автопоезда / В. В. Маланин, В. В. Аюпов // Деп. в НИИН автопром. 一№ 1005ап. 84деп. - 1984.- 13 с .

4. Пасхин Б. Определение маневренных площадей для автомобильных поездов ЗИЛ-5 / Б. Пасхин // Автомобиль.— 194І.— № 3.—C. 17-20.

5. Беккер, М. Г. Введение в теорию систем местность - машина / М. Г. Беккер; пер. с англ. доктора технических наук В. В. Гуськова - М.: Машиностроение, 1973.-Б/C.

6. Терлецкий, В.Г., Тохтарь Г. И. Исследование устойчивости движения автопоезда повышенной проходимости / В. Г. Терлецкий, Г. И. Тохтарь // Изв. вузов: Машиностроение, 1977.— № 2.-С. 94-97.

7. Liu Q. Vehicle lateral position prediction: A small step towards a comprehensive risk assessment system / Q. Liu, B. Lathrop, and V. Butakov // IEEE17th International Conference on Intelligent Transportation Systems (ITSC) - 2014.

8. Morrison, G. Sideslip estimation for articulated heavy vehicles at the limits of adhesion / G. Morrison, D. Cebon // Vehicle System Dynamics. — 2016. — D0I:10.10 80/00423114.2016.1223326. - W/P.

9. Atev S. Clustering of vehicle trajectories / S. Atev, G. Miller, P. Papanikolopoulos // IEEE Transactions on Intelligent Transportation Systems// vol. 11, no. 3-2012.

10. Амосов А. Г. Графоаналитический метод построения траектории движения неделимых грузов: диссертация на соискание ученой степени кандидат технических наук: 05.01.01 / Амосов А. Г.- - М.: 2019.

(c) Амосов Алексей Германович ( lamosov@yandex.ru ), Голиков Владислав Андреевич ( fordik08@mail.ru ). Журнал «Современная наука: актуальные проблемы теории и практики» 